\title{
Characteristics of SARS-CoV-2 and COVID-19
}

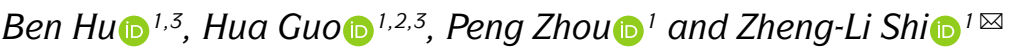

Abstract | Severe acute respiratory syndrome coronavirus 2 (SARS-CoV-2) is a highly transmissible and pathogenic coronavirus that emerged in late 2019 and has caused a pandemic of acute respiratory disease, named 'coronavirus disease 2019' (COVID-19), which threatens human health and public safety. In this Review, we describe the basic virology of SARS-CoV-2, including genomic characteristics and receptor use, highlighting its key difference from previously known coronaviruses. We summarize current knowledge of clinical, epidemiological and pathological features of COVID-19, as well as recent progress in animal models and antiviral treatment approaches for SARS-CoV-2 infection. We also discuss the potential wildlife hosts and zoonotic origin of this emerging virus in detail.

Coronaviruses are a diverse group of viruses infecting many different animals, and they can cause mild to severe respiratory infections in humans. In 2002 and 2012, respectively, two highly pathogenic coronaviruses with zoonotic origin, severe acute respiratory syndrome coronavirus (SARS-CoV) and Middle East respiratory syndrome coronavirus (MERS-CoV), emerged in humans and caused fatal respiratory illness, making emerging coronaviruses a new public health concern in the twenty-first century ${ }^{1}$. At the end of 2019, a novel coronavirus designated as SARS-CoV-2 emerged in the city of Wuhan, China, and caused an outbreak of unusual viral pneumonia. Being highly transmissible, this novel coronavirus disease, also known as coronavirus disease 2019 (COVID-19), has spread fast all over the world ${ }^{2,3}$. It has overwhelmingly surpassed SARS and MERS in terms of both the number of infected people and the spatial range of epidemic areas. The ongoing outbreak of COVID-19 has posed an extraordinary threat to global public health ${ }^{4,5}$. In this Review, we summarize the current understanding of the nature of SARS-CoV-2 and COVID-19. On the basis of recently published findings, this comprehensive Review covers the basic biology of SARS-CoV-2, including the genetic characteristics, the potential zoonotic origin and its receptor binding. Furthermore, we will discuss the clinical and epidemiological features, diagnosis of and countermeasures against COVID-19.

\section{Emergence and spread}

In late December 2019, several health facilities in Wuhan, in Hubei province in China, reported clusters of patients with pneumonia of unknown cause ${ }^{6}$. Similarly to patients with SARS and MERS, these patients showed symptoms of viral pneumonia, including fever, cough and chest discomfort, and in severe cases dyspnea and bilateral lung infiltration ${ }^{6,7}$. Among the first 27 documented hospitalized patients, most cases were epidemiologically linked to Huanan Seafood Wholesale Market, a wet market located in downtown Wuhan, which sells not only seafood but also live animals, including poultry and wildlife $\mathrm{e}^{4,8}$. According to a retrospective study, the onset of the first known case dates back to 8 December 2019 (REF. ${ }^{9}$ ). On 31 December, Wuhan Municipal Health Commission notified the public of a pneumonia outbreak of unidentified cause and informed the World Health Organization (WHO) ${ }^{9}$ (FIG. 1).

By metagenomic RNA sequencing and virus isolation from bronchoalveolar lavage fluid samples from patients with severe pneumonia, independent teams of Chinese scientists identified that the causative agent of this emerging disease is a betacoronavirus that had never been seen before ${ }^{6,10,11}$. On 9 January 2020, the result of this etiological identification was publicly announced (FIG. 1). The first genome sequence of the novel coronavirus was published on the Virological website on 10 January, and more nearly complete genome sequences determined by different research institutes were then released via the GISAID database on 12 January $^{7}$. Later, more patients with no history of exposure to Huanan Seafood Wholesale Market were identified. Several familial clusters of infection were reported, and nosocomial infection also occurred in health-care facilities. All these cases provided clear evidence for human-to-human transmission of the new virus ${ }^{4,12-14}$. As the outbreak coincided with the approach of the lunar New Year, travel between cities before the festival facilitated virus transmission in China. This novel coronavirus pneumonia soon spread to other cities in Hubei province and to other parts of China. Within 1 month, 


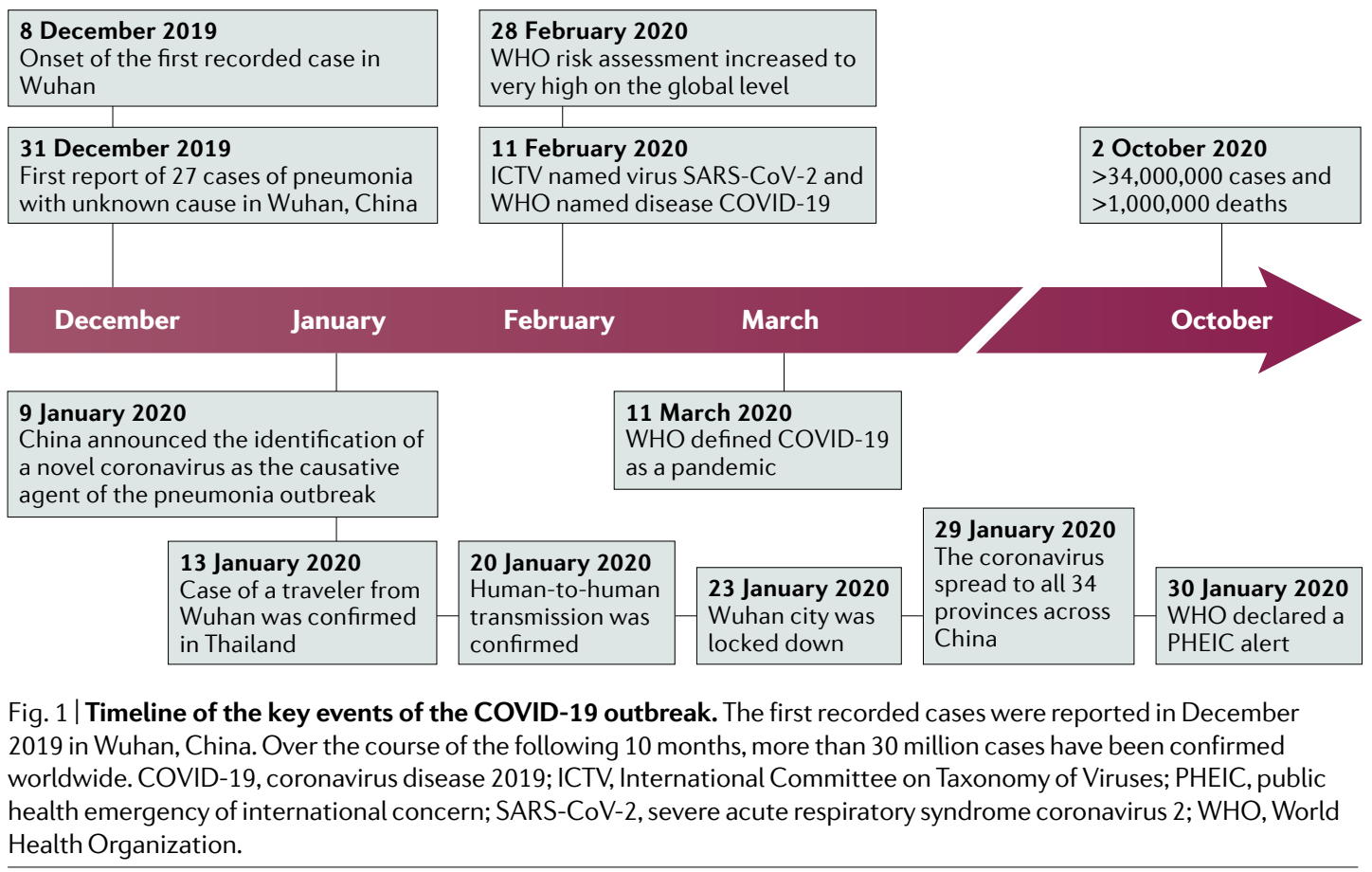

it had spread massively to all 34 provinces of China. The number of confirmed cases suddenly increased, with thousands of new cases diagnosed daily during late January ${ }^{15}$. On 30 January, the WHO declared the novel coronavirus outbreak a public health emergency of international concern ${ }^{16}$. On 11 February, the International Committee on Taxonomy of Viruses named the novel coronavirus 'SARS-CoV-2', and the WHO named the disease 'COVID-19' (REF. $\left.{ }^{17}\right)$.

The outbreak of COVID-19 in China reached an epidemic peak in February. According to the National Health Commission of China, the total number of cases continued to rise sharply in early February at an average rate of more than 3,000 newly confirmed cases per day. To control COVID-19, China implemented unprecedentedly strict public health measures. The city of Wuhan was shut down on 23 January, and all travel and transportation connecting the city was blocked. In the following couple of weeks, all outdoor activities and gatherings were restricted, and public facilities were closed in most cities as well as in countryside ${ }^{18}$. Owing to these measures, the daily number of new cases in China started to decrease steadily ${ }^{19}$.

However, despite the declining trend in China, the international spread of COVID-19 accelerated from late February. Large clusters of infection have been reported from an increasing number of countries ${ }^{18}$. The high transmission efficiency of SARS-CoV-2 and the abundance of international travel enabled rapid worldwide spread of COVID-19. On 11 March 2020, the WHO officially characterized the global COVID-19 outbreak as a pandemic ${ }^{20}$. Since March, while COVID-19 in China has become effectively controlled, the case numbers in Europe, the USA and other regions have jumped sharply. According to the COVID-19 dashboard of the Center for System Science and Engineering at Johns Hopkins University, as of 11 August 2020,
216 countries and regions from all six continents had reported more than 20 million cases of COVID-19, and more than 733,000 patients had died ${ }^{21}$. High mortality occurred especially when health-care resources were overwhelmed. The USA is the country with the largest number of cases so far.

Although genetic evidence suggests that SARS-CoV-2 is a natural virus that likely originated in animals, there is no conclusion yet about when and where the virus first entered humans. As some of the first reported cases in Wuhan had no epidemiological link to the seafood market ${ }^{22}$, it has been suggested that the market may not be the initial source of human infection with SARS-CoV-2. One study from France detected SARS-CoV- 2 by PCR in a stored sample from a patient who had pneumonia at the end of 2019, suggesting SARS-CoV-2 might have spread there much earlier than the generally known starting time of the outbreak in France ${ }^{23}$. However, this individual early report cannot give a solid answer to the origin of SARS-CoV-2 and contamination, and thus a false positive result cannot be excluded. To address this highly controversial issue, further retrospective investigations involving a larger number of banked samples from patients, animals and environments need to be conducted worldwide with well-validated assays.

\section{Genomics, phylogeny and taxonomy}

As a novel betacoronavirus, SARS-CoV-2 shares $79 \%$ genome sequence identity with SARS-CoV and $50 \%$ with MERS- $\mathrm{CoV}^{24}$. Its genome organization is shared with other betacoronaviruses. The six functional open reading frames (ORFs) are arranged in order from $5^{\prime}$ to $3^{\prime}$ : replicase (ORF1a/ORF1b), spike (S), envelope $(\mathrm{E})$, membrane $(\mathrm{M})$ and nucleocapsid $(\mathrm{N})$. In addition, seven putative ORFs encoding accessory proteins are interspersed between the structural genes ${ }^{25}$. Most of the proteins encoded by SARS-CoV-2 have a similar 
length to the corresponding proteins in SARS-CoV. Of the four structural genes, SARS-CoV-2 shares more than $90 \%$ amino acid identity with SARS-CoV except for the $S$ gene, which diverges ${ }^{11,24}$. The replicase gene covers two thirds of the $5^{\prime}$ genome, and encodes a large polyprotein (pplab), which is proteolytically cleaved into 16 non-structural proteins that are involved in transcription and virus replication. Most of these SARS-CoV-2 non-structural proteins have greater than $85 \%$ amino acid sequence identity with SARS-CoV ${ }^{25}$.

The phylogenetic analysis for the whole genome shows that SARS-CoV-2 is clustered with SARS-CoV and SARS-related coronaviruses (SARSr-CoVs) found in bats, placing it in the subgenus Sarbecovirus of the genus Betacoronavirus. Within this clade, SARS-CoV-2 is grouped in a distinct lineage together with four horseshoe bat coronavirus isolates (RaTG13, RmYN02, ZC45 and ZXC21) as well as novel coronaviruses recently identified in pangolins, which group parallel to SARS-CoV and other SARSr-CoVs (FIG. 2). Using sequences of five conserved replicative domains in pplab (3C-like protease (3CLpro), nidovirus RNA-dependent RNA polymerase (RdRp)-associated nucleotidyltransferase (NiRAN), RdRp, zinc-binding domain (ZBD) and HEL1), the Coronaviridae Study Group of the International Committee on Taxonomy of Viruses estimated the pairwise patristic distances between SARS-CoV-2 and known coronaviruses, and assigned SARS-CoV-2 to the existing species SARSr-CoV ${ }^{17}$. Although phylogenetically related, SARS-CoV-2 is distinct from all other coronaviruses from bats and pangolins in this species.

The SARS-CoV-2 S protein has a full size of 1,273 amino acids, longer than that of SARS-CoV $(1,255$ amino acids) and known bat SARSr-CoVs (1,245-1,269 amino acids). It is distinct from the S proteins of most members in the subgenus Sarbecovirus, sharing amino acid sequence similarities of 76.777.0\% with SARS-CoVs from civets and humans,

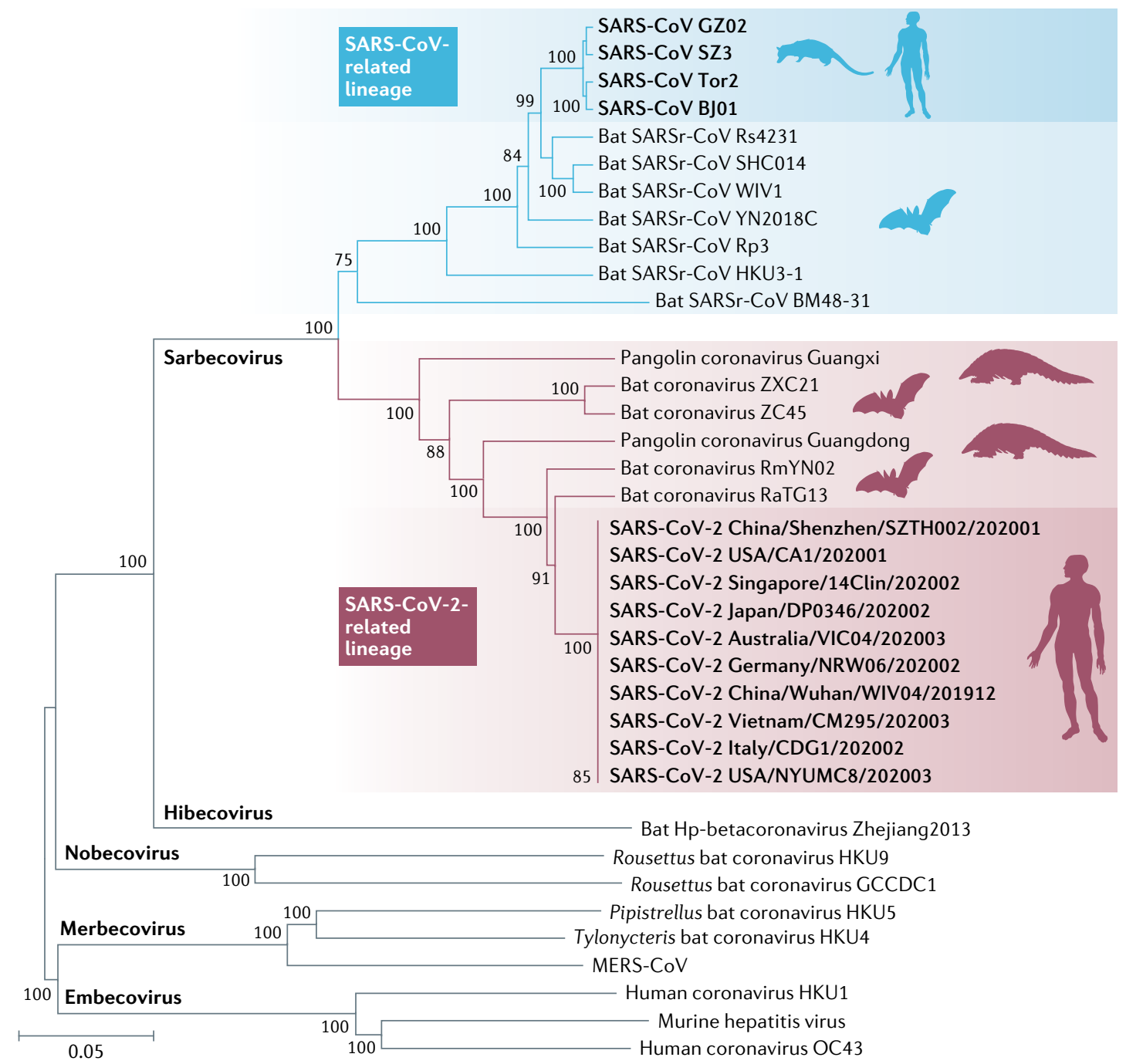

Fig. 2 | Phylogenetic tree of the full-length genome sequences of SARS-CoV-2, SARSr-CoVs and other betacoronaviruses. The construction was performed by the neighbour joining method with use of the program MEGA6 with bootstrap values being calculated from 1,000 trees. Severe acute respiratory syndrome coronavirus 2 (SARS-CoV-2) clusters with closely related viruses in bats and pangolins and together with SARS-CoV and bat SARS-related coronaviruses (SARSr-CoVs) forms the sarbecoviruses. The sequences were downloaded from the GISAID database and GenBank. MERS-CoV, Middle East respiratory syndrome coronavirus. 
$75-97.7 \%$ with bat coronaviruses in the same subgenus and $90.7-92.6 \%$ with pangolin coronaviruses ${ }^{11}$. In the receptor-binding domain (RBD) of $S$ protein, the amino acid similarity between SARS-CoV-2 and
SARS-CoV is only 73\%. Another specific genomic feature of SARS-CoV-2 is the insertion of four amino acid residues (PRRA) at the junction of subunits $S 1$ and $\mathrm{S} 2$ of the S protein ${ }^{26}$ (FIG. 3a). This insertion generates

$\overbrace{\text { NTD }}^{100}$

\section{SARS-CoV-2}

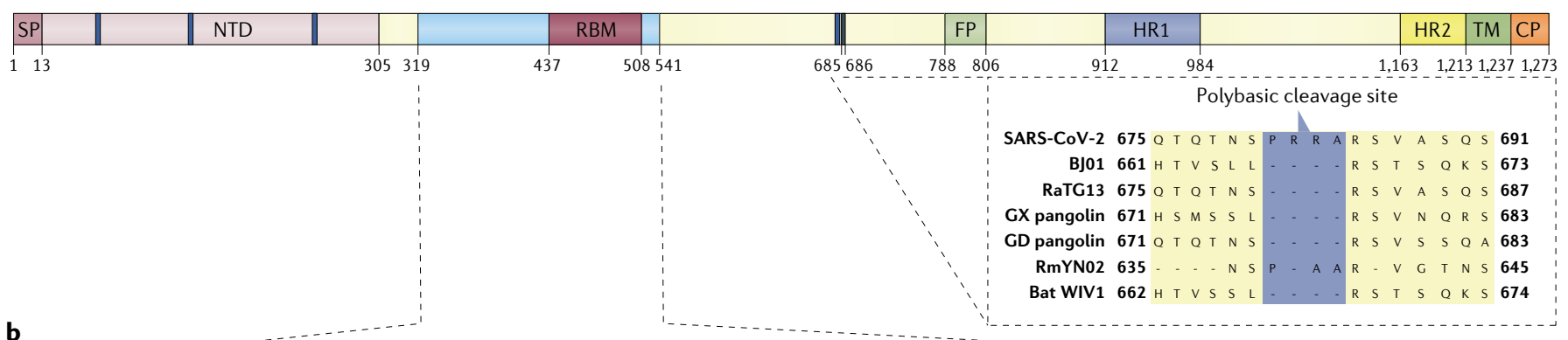

b

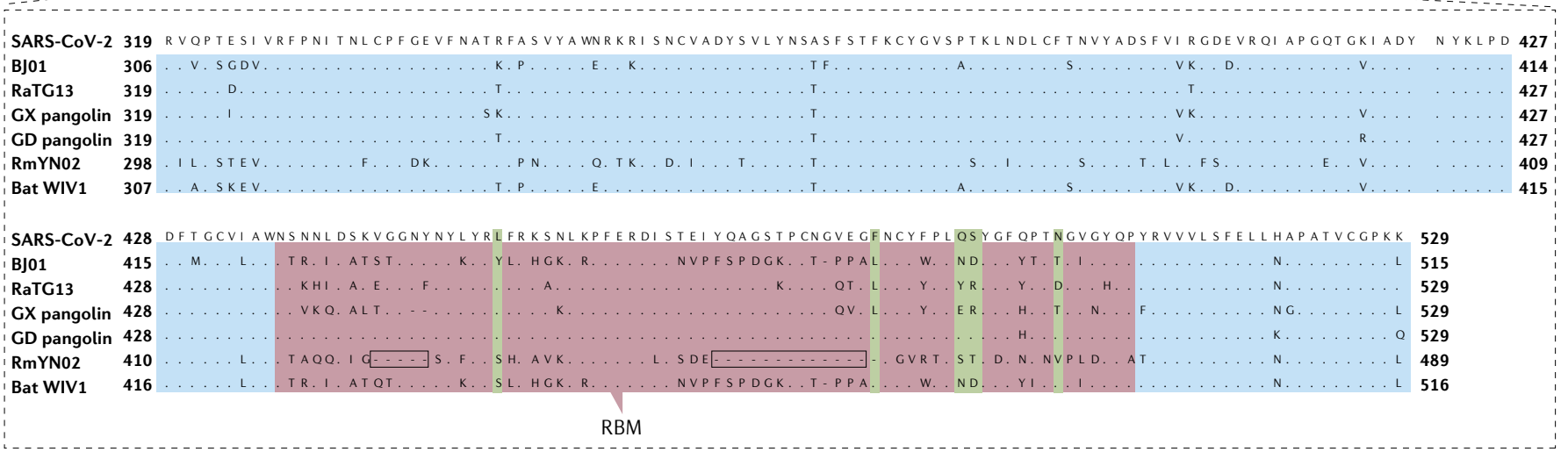

\begin{tabular}{lllllll} 
RBD & Host & \multicolumn{5}{c}{ Important residues in RBD contact with ACE2 } \\
\cline { 3 - 7 } & & 445 & 486 & 493 & 494 & 501 \\
\hline SARS-CoV-2 & Human & $\mathrm{L}$ & $\mathrm{F}$ & $\mathrm{Q}$ & $\mathrm{S}$ & $\mathrm{N}$ \\
\hline SARS-CoV & Human & $\mathrm{Y}(442)$ & $\mathrm{L}(472)$ & $\mathrm{N}(479)$ & $\mathrm{D}(480)$ & $\mathrm{T}(487)$ \\
\hline RaTG13 & Rhinolophus affinis & $\mathrm{L}$ & $\mathrm{L}$ & $\mathrm{Y}$ & $\mathrm{R}$ & $\mathrm{N}$ \\
GX pangolin & Pangolin & $\mathrm{L}$ & $\mathrm{L}$ & $\mathrm{E}$ & $\mathrm{R}$ & $\mathrm{T}$ \\
GD pangolin & Pangolin & $\mathrm{L}$ & $\mathrm{F}$ & $\mathrm{Q}$ & $\mathrm{S}$ & $\mathrm{N}$ \\
\hline RmYN02 & Rhinolophus malayanus S(429) & - & $\mathrm{S}(453)$ & $\mathrm{T}(454)$ & $\mathrm{V}(461)$ \\
\hline Bat WIV1 & Rhinolophus sinicus & $\mathrm{S}(443)$ & $\mathrm{F}(473)$ & $\mathrm{N}(480)$ & $\mathrm{D}(481)$ & $\mathrm{N}(488)$ \\
\hline
\end{tabular}

d

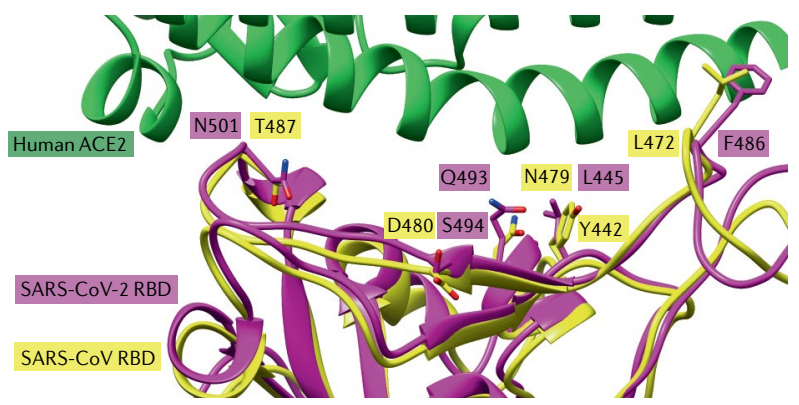

Fig. 3 | Key differences in the spike protein of SARS-CoV-2 and related coronaviruses. a | Schematic diagram of the spike (S) protein of severe acute respiratory syndrome coronavirus (SARS-CoV) and SARS-CoV-2. The residue numbers of each region correspond to their positions in the $S$ proteins of SARS-CoV and SARS-CoV-2. The dark blue blocks represent insertions in the S protein. The insertions at amino acids $675-691$ of the SARS-CoV-2 S protein are shown in an enlargement at the bottom right and aligned with those of other coronaviruses in the same region. $\mathbf{b} \mid$ Alignment of the receptor-binding domain (RBD) in SARS-CoV-2, SARS-CoV BJ01, RaTG13, pangolin coronavirus reported from Guangdong, China (GD pangolin), pangolin coronavirus reported from Guangxi, China (GX pangolin) and bat SARS-related coronavirus (SARSr-CoV) WIV1. The receptor-binding motif (RBM) is shown in purple, and the five key residues that contact angiotensin-converting enzyme 2 (ACE2) directly are highlighted in green. c | Five critical residues in the RBD of SARS-CoV-2 and other viruses. d / Comparison of the structure of SARS-CoV-2 and SARS-CoV RBD complexed with human ACE2 (hACE2); SARS-CoV-2 RBM in purple, SARS-CoV RBM in yellow and hACE2 in green). Five critical residues that are involved in the RBM-ACE2 binding are shown. The Protein Data Bank codes are 2AJF for SARS-CoV RBD-hACE2 and 6VW1 for SARS-CoV-2 RBD-hACE2. The GenBank entries are AY278488 for SARS-CoV BJ01, MN996532 for the bat SARSr-CoV RaTG13, MT121216 and MT072864 for the GD pangolin and GX pangolin coronaviruses, respectively, and KF367457 for the bat SARSr-CoV WIV1. CP, cytoplasmic domain; FP, fusion peptide; HR1, heptad repeat 1; HR2, heptad repeat 2; NTD, $\mathrm{N}$-terminal domain; SP, signal peptide; TM, transmembrane domain. Parts a and $\mathbf{b}$ adapted from REF. ${ }^{26}$, Springer Nature Limited. 
a polybasic cleavage site (RRAR), which enables effective cleavage by furin and other proteases ${ }^{27}$. Such an S1-S2 cleavage site is not observed in all related viruses belonging to the subgenus Sarbecovirus, except for a similar three amino acid insertion (PAA) in RmYN02, a bat-derived coronavirus newly reported from Rhinolophus malayanus in China ${ }^{28}$ (FIG. 3a). Although the insertion in RmYN02 does not functionally represent a polybasic cleavage site, it provides support for the notion that this characteristic, initially considered unique to SARS-CoV-2, has been acquired naturally ${ }^{28}$. A structural study suggested that the furin-cleavage site can reduce the stability of SARS-CoV-2 S protein and facilitate the conformational adaption that is required for the binding of the RBD to its receptor ${ }^{29}$. Whether the higher transmissibility of SARS-CoV-2 compared with SARS-CoV is a gain of function associated with acquisition of the furin-like cleavage site is yet to be demonstrated ${ }^{26}$.

An additional distinction is the accessory gene orf8 of SARS-CoV-2, which encodes a novel protein showing only $40 \%$ amino acid identity to ORF8 of SARS-CoV. Unlike in SARS-CoV, this new ORF8 protein does not contain a motif that triggers intracellular stress pathways $^{25}$. Notably, a SARS-CoV-2 variant with a 382-nucleotide deletion covering the whole of ORF8 has been discovered in a number of patients in Singapore, which resembles the 29- or 415-nucleotide deletions in the ORF8 region observed in human SARS-CoV variants from the late phase of the 2002-2003 outbreak ${ }^{30}$. Such ORF8 deletion may be indicative of human adaptation after cross-species transmission from an animal host.

To assess the genetic variation of different SARSCoV-2 strains, the 2019 Novel Coronavirus Resource of China National Center for Bioinformation aligned 77,801 genome sequences of SARS-CoV-2 detected globally and identified a total of 15,018 mutations, including 14,824 single-nucleotide polymorphisms (BIGD) ${ }^{31}$. In the $\mathrm{S}$ protein, four amino acid alterations, V483A, L455I, F456V and G476S, are located near the binding interface in the RBD, but their effects on binding to the host receptor are unknown. The alteration D614G in the $S 1$ subunit was found far more frequently than other $S$ variant sites, and it is the marker of a major subclade of SARS-CoV-2 (clade G). Since March 2020, SARS-CoV-2 variants with G614 in the S protein have replaced the original D614 variants and become the dominant form circulating globally. Compared with the D614 variant, higher viral loads were found in patients infected with the G614 variant, but clinical data suggested no significant link between the D614G alteration and disease severity $^{32}$. Pseudotyped viruses carrying the $S$ protein with G614 generated higher infectious titres than viruses carrying the S protein with D614, suggesting the alteration may have increased the infectivity of SARS-CoV-2 $\left(\mathrm{REF}^{32}\right)$. However, the results of in vitro experiments based on pseudovirus models may not exactly reflect natural infection. This preliminary finding should be validated by more studies using wild-type SARS-CoV-2 variants to infect different target cells and animal models. Whether this amino acid change enhanced virus transmissibility is also to be determined. Another marker mutation for SARS-CoV-2 evolution is the single-nucleotide polymorphism at nucleotide position 28,144 , which results in amino acid substitution of Ser for Lys at residue 84 of the ORF8 protein. Those variants with this mutation make up a single subclade labelled as 'clade $S^{33,34}$. Currently, however, the available sequence data are not sufficient to interpret the early global transmission history of the virus, and travel patterns, founder effects and public health measures also strongly influence the spread of particular lineages, irrespective of potential biological differences between different virus variants.

\section{Animal host and spillover}

Bats are important natural hosts of alphacoronaviruses and betacoronaviruses. The closest relative to SARS-CoV-2 known to date is a bat coronavirus detected in Rhinolophus affinis from Yunnan province, China, named 'RaTG13', whose full-length genome sequence is $96.2 \%$ identical to that of SARS-CoV-2 (REF. ${ }^{11}$ ). This bat virus shares more than $90 \%$ sequence identity with SARS-CoV-2 in all ORFs throughout the genome, including the highly variable $S$ and ORF8 $\left(\right.$ REF $^{11}{ }^{11}$. Phylogenetic analysis confirms that SARS-CoV-2 closely clusters with RaTG13 (FIG. 2). The high genetic similarity between SARS-CoV-2 and RaTG13 supports the hypothesis that SARS-CoV-2 likely originated from bats ${ }^{35}$. Another related coronavirus has been reported more recently in a Rhinolophus malayanus bat sampled in Yunnan. This novel bat virus, denoted 'RmYN02', is $93.3 \%$ identical to SARS-CoV-2 across the genome. In the long $1 a b$ gene, it exhibits $97.2 \%$ identity to SARS-CoV-2, which is even higher than for RaTG13 (REF. ${ }^{28}$. In addition to RaTG13 and RmYN02, phylogenetic analysis shows that bat coronaviruses ZC45 and ZXC21 previously detected in Rhinolophus pusillus bats from eastern China also fall into the SARS-CoV-2 lineage of the subgenus Sarbecovirus ${ }^{36}$ (FIG. 2). The discovery of diverse bat coronaviruses closely related to SARS-CoV-2 suggests that bats are possible reservoirs of SARS-CoV-2 $\left(\right.$ REF. $\left.^{37}\right)$. Nevertheless, on the basis of current findings, the divergence between SARS-CoV-2 and related bat coronaviruses likely represents more than 20 years of sequence evolution, suggesting that these bat coronaviruses can be regarded only as the likely evolutionary precursor of SARS-CoV-2 but not as the direct progenitor of SARS-CoV-2 $\left(\mathrm{REF}^{38}\right)$.

Beyond bats, pangolins are another wildlife host probably linked with SARS-CoV-2. MultipleSARS-CoV-2related viruses have been identified in tissues of Malayan pangolins smuggled from Southeast Asia into southern China from 2017 to 2019. These viruses from pangolins independently seized by Guangxi and Guangdong provincial customs belong to two distinct sublineages ${ }^{39-41}$. The Guangdong strains, which were isolated or sequenced by different research groups from smuggled pangolins, have $99.8 \%$ sequence identity with each other $^{41}$. They are very closely related to SARS-CoV-2, exhibiting $92.4 \%$ sequence similarity. Notably, the RBD of Guangdong pangolin coronaviruses is highly similar to that of SARS-CoV-2. The receptor-binding motif (RBM; which is part of the RBD) of these viruses has only one amino acid variation from SARS-CoV-2, and it is identical to that of SARS-CoV-2 in all five critical 
residues for receptor binding ${ }^{40}$ (FIG. 3b). In comparison with the Guangdong strains, pangolin coronaviruses reported from Guangxi are less similar to SARS-CoV-2, with $85.5 \%$ genome sequence identity ${ }^{39}$. The repeated occurrence of SARS-CoV-2-related coronavirus infections in pangolins from different smuggling events suggests that these animals are possible hosts of the viruses. However, unlike bats, which carry coronaviruses healthily, the infected pangolins showed clinical signs and histopathological changes, including interstitial pneumonia and inflammatory cell infiltration in diverse organs $s^{40}$. These abnormalities suggest that pangolins are unlikely to be the reservoir of these coronaviruses but more likely acquired the viruses after spillover from the natural hosts.

An intermediate host usually plays an important role in the outbreak of bat-derived emerging coronaviruses; for example, palm civets for SARS-CoV and dromedary camels for MERS-CoV. The virus strains carried by these two intermediate hosts were almost genetically identical to the corresponding viruses in humans (more than $99 \%$ genome sequence identity) ${ }^{1}$. Despise an RBD that is virtually identical to that of SARS-CoV-2, the pangolin coronaviruses known to date have no more than $92 \%$ genome identity with SARS-CoV-2 (REF. ${ }^{42}$ ). The available data are insufficient to interpret pangolins as the intermediate host of SARS-CoV-2. So far, no evidence has shown that pangolins were directly involved in the emergence of SARS-CoV-2.

Currently, our knowledge on the animal origin of SARS-CoV-2 remains incomplete to a large part. The reservoir hosts of the virus have not been clearly proven. It is unknown whether SARS-CoV-2 was transmitted to humans through an intermediate host and which animals may act as its intermediate host. Detection of RaTG13, RmYN02 and pangolin coronaviruses implies that diverse coronaviruses similar to SARS-CoV-2 are circulating in wildlife. In addition, as previous studies showed recombination as the potential origin of some sarbecoviruses such as SARS-CoV, it cannot be excluded that viral RNA recombination among different related coronaviruses was involved in the evolution of SARS-CoV-2. Extensive surveillance of SARS-CoV-2related viruses in China, Southeast Asia and other regions targeting bats, wild and captured pangolins and other wildlife species will help us to better understand the zoonotic origin of SARS-CoV-2.

Besides wildlife, researchers investigated the susceptibility of domesticated and laboratory animals to SARS-CoV-2 infection. The study demonstrated experimentally that SARS-CoV-2 replicates efficiently in cats and in the upper respiratory tract of ferrets, whereas dogs, pigs, chickens and ducks were not susceptible to SARS-CoV-2 (REF. $\left.{ }^{43}\right)$. The susceptibility of minks was documented by a report from the Netherlands on an outbreak of SARS-CoV-2 infection in farmed minks. Although the symptoms in most infected minks were mild, some developed severe respiratory distress and died of interstitial pneumonia ${ }^{44}$. Both virological and serological testing found evidence for natural SARS-CoV-2 infection in two dogs from households with human cases of COVID-19 in Hong Kong, but the dogs appeared asymptomatic ${ }^{45}$. Another serological study detected SARS-CoV-2 neutralizing antibodies in cat serum samples collected in Wuhan after the COVID-19 outbreak, providing evidence for SARS-CoV-2 infection in cat populations in Wuhan, although the potential of SARS-CoV-2 transmission from cats to humans is currently uncertain ${ }^{46}$.

\section{Receptor use and pathogenesis}

SARS-CoV-2 uses the same receptor as SARS-CoV, angiotensin-converting enzyme 2 (ACE2) $)^{11,47}$. Besides human ACE2 (hACE2), SARS-CoV-2 also recognizes ACE2 from pig, ferret, rhesus monkey, civet, cat, pangolin, rabbit and $\operatorname{dog}^{11,43,48,49}$. The broad receptor usage of SARS-CoV-2 implies that it may have a wide host range, and the varied efficiency of ACE2 usage in different animals may indicate their different susceptibilities to SARS-CoV-2 infection. The S1 subunit of a coronavirus is further divided into two functional domains, an $\mathrm{N}$-terminal domain and a $\mathrm{C}$-terminal domain. Structural and biochemical analyses identified a 211 amino acid region (amino acids 319-529) at the S1 C-terminal domain of SARS-CoV-2 as the RBD, which has a key role in virus entry and is the target of neutralizing antibodies ${ }^{50,51}$ (FIC. 3a). The RBM mediates contact with the ACE2 receptor (amino acids 437-507 of SARS-CoV-2 S protein), and this region in SARS-CoV-2 differs from that in SARS-CoV in the five residues critical for ACE2 binding, namely Y455L, L486F, N493Q, D494S and T501N ${ }^{52}$ (FIG. 3b,c). Owing to these residue changes, interaction of SARS-CoV-2 with its receptor stabilizes the two virus-binding hotspots on the surface of hACE2 (REF. ${ }^{50}$ ) (FIG. 3d). Moreover, a four-residue motif in the RBM of SARS-CoV-2 (amino acids 482-485: G-V-E-G) results in a more compact conformation of its hACE2-binding ridge than in SARS-CoV and enables better contact with the N-terminal helix of hACE2 $\left(\right.$ REF $\left.^{50}\right)$. Biochemical data confirmed that the structural features of the SARS-CoV-2 RBD has strengthened its hACE2 binding affinity compared with that of SARS-CoV ${ }^{50,52,53}$.

Similarly to other coronaviruses, SARS-CoV-2 needs proteolytic processing of the $S$ protein to activate the endocytic route. It has been shown that host proteases participate in the cleavage of the $S$ protein and activate the entry of SARS-CoV-2, including transmembrane protease serine protease 2 (TMPRSS2), cathepsin L and furin $^{47,54,55}$. Single-cell RNA sequencing data showed that TMPRSS2 is highly expressed in several tissues and body sites and is co-expressed with ACE2 in nasal epithelial cells, lungs and bronchial branches, which explains some of the tissue tropism of SARS-CoV-2 (REFS ${ }^{56,57}$ ). SARS-CoV-2 pseudovirus entry assays revealed that TMPRSS2 and cathepsin L have cumulative effects with furin on activating virus entry ${ }^{55}$. Analysis of the cryo-electron microscopy structure of SARS-CoV-2 S protein revealed that its RBD is mostly in the lying-down state, whereas the SARS-CoV S protein assumes equally standing-up and lying-down conformational states ${ }^{50,51,58,59}$. A lying-down conformation of the SARS-CoV-2 $S$ protein may not be in favour of receptor binding but is helpful for immune evasion ${ }^{55}$. 
The pathogenesis of SARS-CoV-2 infection in humans manifests itself as mild symptoms to severe respiratory failure. On binding to epithelial cells in the respiratory tract, SARS-CoV-2 starts replicating and migrating down to the airways and enters alveolar epithelial cells in the lungs. The rapid replication of SARS-CoV-2 in the lungs may trigger a strong immune response. Cytokine storm syndrome causes acute respiratory distress syndrome and respiratory failure, which is considered the main cause of death in patients with COVID-19 (REFS ${ }^{60,61}$ ). Patients of older age ( $>60$ years) and with serious pre-existing diseases have a greater risk of developing acute respiratory distress syndrome and death $^{62-64}$ (FIG. 4). Multiple organ failure has also been reported in some COVID-19 cases $^{9,13,65}$.

Histopathological changes in patients with COVID-19 occur mainly in the lungs. Histopathology analyses showed bilateral diffused alveolar damage, hyaline membrane formation, desquamation of pneumocytes and fibrin deposits in lungs of patients with severe COVID-19. Exudative inflammation was also shown in some cases. Immunohistochemistry assays detected SARS-CoV-2 antigen in the upper airway, bronchiolar epithelium and submucosal gland epithelium, as well as in type I and type II pneumocytes, alveolar macrophages and hyaline membranes in the lungs ${ }^{13,60,66,67}$.

Animal models used for studying SARS-CoV-2 infection pathogenesis include non-human primates (rhesus macaques, cynomolgus monkeys, marmosets and African green monkeys), mice (wild-type mice (with mouse-adapted virus) and human ACE2-transgenic or human ACE2-knock-in mice), ferrets and golden hamsters ${ }^{43,48,68-74}$. In non-human primate animal models, most species display clinical features similar to those of patients with COVID-19, including virus shedding, virus replication and host responses to SARS-CoV-2 infection ${ }^{69,72,73}$. For example, in the rhesus macaque model, high viral loads were detected in the upper and lower respiratory tracts. Acute viral interstitial pneumonia and humoral and cellular immune responses were observed ${ }^{48,75}$. Moreover, prolonged virus shedding peaked early in the course of infection in asymptomatic macaques ${ }^{69}$, and old monkeys showed severer interstitial pneumonia than young monkeys ${ }^{76}$, which is similar to what is seen in patients with COVID-19. In human ACE2-transgenic mice infected with SARS-CoV-2, typical interstitial pneumonia was present, and viral antigens were observed mainly in the bronchial epithelial cells, macrophages and alveolar epithelia. Some human ACE2-transgenic mice even died after infection ${ }^{70,71}$. In wide-type mice, a SARS-CoV-2 mouse-adapted strain with the N501Y alteration in the RBD of the S protein was generated at passage 6 . Interstitial pneumonia and inflammatory responses were found in both young and aged mice after infection with the mouse-adapted $\operatorname{strain}^{74}$. Golden hamsters also showed typical symptoms after being infected with SARS-CoV-2 (REF. ${ }^{77}$ ). In other animal models, including cats and ferrets, SARS-CoV-2 could efficiently replicate in the upper respiratory tract but did not induce severe clinical symptoms ${ }^{43,78}$. As transmission by direct contact and air was observed in infected ferrets and hamsters, these animals could be used to model different transmission modes of COVID-19 $\left(\mathrm{REFS}^{77-79}\right)$. Animal models offer important information for understanding the pathogenesis of SARS-CoV-2 infection and the transmission dynamics of SARS$\mathrm{CoV}-2$, and are important to evaluate the efficacy of antiviral therapeutics and vaccines.

\section{Clinical and epidemiological features}

It appears that all ages of the population are susceptible to SARS-CoV-2 infection, and the median age of infection is around 50 years ${ }^{9,13,60,80,81}$. However, clinical manifestations differ with age. In general, older men ( $>60$ years old) with co-morbidities are more likely to develop severe respiratory disease that requires hospitalization

Age as major risk factor

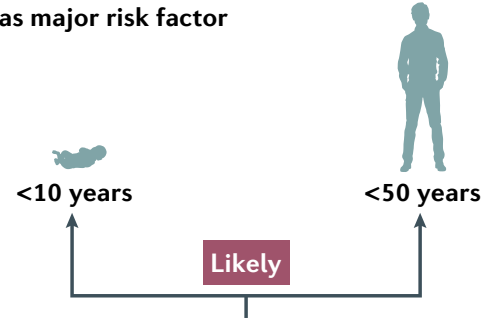

COVID-19 cases (percentage of all cases)

\begin{tabular}{|c|c|c|c|}
\hline Incubation period & $\begin{array}{l}\text { - Fever, fatigue and dry cough } \\
\text { - Ground-glass opacities } \\
\text { - Pneumonia }\end{array}$ & $\begin{array}{l}\text { - Dyspnea } \\
\text { - Coexisting illness } \\
\text { - ICU needed }\end{array}$ & $\begin{array}{l}\text { - ARDS } \\
\text { - Acute cardiac injury } \\
\text { - Multi-organ failure }\end{array}$ \\
\hline $\begin{array}{c}\sim 5 \text { days } \\
(1-14)\end{array}$ & onset & & $\begin{array}{l}\sim 16 \text { days } \\
(12-20)\end{array}$ \\
\hline
\end{tabular}

Fig. 4 | Clinical features of COVID-19. Typical symptoms of coronavirus disease 2019 (COVID-19) are fever, dry cough and fatigue and in severer cases dyspnea. Many infections, in particular in children and young adults, are asymptomatic, whereas older people and/or people with co-morbidities are at higher risk of severe disease, respiratory failure and death. The incubation period is $\sim 5$ days, severe disease usually develops $\sim 8$ days after symptom onset and critical disease and death occur at $\sim 16$ days. ARDS, acute respiratory distress syndrome; ICU, intensive care unit. 
or even die, whereas most young people and children have only mild diseases (non-pneumonia or mild pneumonia) or are asymptomatic ${ }^{9,81,82}$. Notably, the risk of disease was not higher for pregnant women. However, evidence of transplacental transmission of SARS-CoV-2 from an infected mother to a neonate was reported, although it was an isolated case ${ }^{83,84}$. On infection, the most common symptoms are fever, fatigue and dry cough $^{13,60,80,81}$. Less common symptoms include sputum production, headache, haemoptysis, diarrhoea, anorexia, sore throat, chest pain, chills and nausea and vomiting in studies of patients in China ${ }^{13,60,80,81}$. Self-reported olfactory and taste disorders were also reported by patients in Italy ${ }^{85}$. Most people showed signs of diseases after an incubation period of 1-14 days (most commonly around 5 days), and dyspnoea and pneumonia developed within a median time of 8 days from illness onset ${ }^{9}$.

In a report of 72,314 cases in China, $81 \%$ of the cases were classified as mild, $14 \%$ were severe cases that required ventilation in an intensive care unit (ICU) and a $5 \%$ were critical (that is, the patients had respiratory failure, septic shock and/or multiple organ dysfunction or failure $)^{9,86}$. On admission, ground-glass opacity was the most common radiologic finding on chest computed tomography $(\mathrm{CT})^{13,60,80,81}$. Most patients also developed marked lymphopenia, similar to what was observed in patients with SARS and MERS, and non-survivors developed severer lymphopenia over time $e^{13,60,80,81}$. Compared with non-ICU patients, ICU patients had higher levels of plasma cytokines, which suggests an immunopathological process caused by a cytokine storm ${ }^{60,86,87}$. In this cohort of patient, around $2.3 \%$ people died within a median time of 16 days from disease onset ${ }^{9,86}$. Men older than 68 years had a higher risk of respiratory failure, acute cardiac injury and heart failure that led to death, regardless of a history of cardiovascular disease ${ }^{86}$ (FIG. 4). Most patients recovered enough to be released from hospital in 2 weeks ${ }^{9,80}$ (FIG. 4).

Early transmission of SARS-CoV-2 in Wuhan in December 2019 was initially linked to the Huanan Seafood Wholesale Market, and it was suggested as the source of the outbreak ${ }^{9,22,60}$. However, community transmission might have happened before that ${ }^{88}$. Later, ongoing human-to-human transmission propagated the outbreak ${ }^{9}$. It is generally accepted that SARS-CoV-2 is more transmissible than SARS-CoV and MERS-CoV; however, determination of an accurate reproduction number (R0) for COVID-19 is not possible yet, as many asymptomatic infections cannot be accurately accounted for at this stage ${ }^{89}$. An estimated R0 of 2.5 (ranging from 1.8 to 3.6) has been proposed for SARS-CoV-2 recently, compared with 2.0-3.0 for SARS-CoV ${ }^{90}$. Notably, most of the SARS-CoV-2 human-to-human transmission early in China occurred in family clusters, and in other countries large outbreaks also happened in other settings, such as migrant worker communities, slaughterhouses and meat packing plants, indicating the necessity of isolating infected people $e^{9,12,91-93}$. Nosocomial transmission was not the main source of transmission in China because of the implementation of infection control measures in clinical settings ${ }^{9}$. By contrast, a high risk of nosocomial transmission was reported in some other areas. For example, a cohort study in London revealed $44 \%$ of the frontline health-care workers from a hospital were infected with SARS-CoV-2 (REF. $\left.{ }^{94}\right)$.

The high transmissibility of SARS-CoV-2 may be attributed to the unique virological features of SARS-CoV-2. Transmission of SARS-CoV occurred mainly after illness onset and peaked following disease severity ${ }^{95}$. However, the SARS-CoV-2 viral load in upper respiratory tract samples was already highest during the first week of symptoms, and thus the risk of pharyngeal virus shedding was very high at the beginning of infection ${ }^{96,97}$. It was postulated that undocumented infections might account for $79 \%$ of documented cases owing to the high transmissibility of the virus during mild disease or the asymptomatic period $^{89}$. A patient with COVID-19 spreads viruses in liquid droplets during speech. However, smaller and much more numerous particles known as aerosol particles can also be visualized, which could linger in the air for a long time and then penetrate deep into the lungs when inhaled by someone else ${ }^{98-100}$. Airborne transmission was also observed in the ferret experiments mentioned above. SARS-CoV-2-infected ferrets shed viruses in nasal washes, saliva, urine and faeces for up to 8 days after infection, and a few naive ferrets with only indirect contact were positive for viral RNA, suggesting airborne transmission ${ }^{78}$. In addition, transmission of the virus through the ocular surface and prolonged presence of SARS-CoV-2 viral RNA in faecal samples were also documented ${ }^{101,102}$. Coronaviruses can persist on inanimate surfaces for days, which could also be the case for SARS-CoV-2 and could pose a prolonged risk of infection ${ }^{103}$. These findings explain the rapid geographic spread of COVID-19, and public health interventions to reduce transmission will provide benefit to mitigate the epidemic, as has proved successful in China and several other countries, such as South Korea ${ }^{89,104,105}$.

\section{Diagnosis}

Early diagnosis is crucial for controlling the spread of COVID-19. Molecular detection of SARS-CoV-2 nucleic acid is the gold standard. Many viral nucleic acid detection kits targeting ORF1b (including RdRp), N, E or $S$ genes are commercially available ${ }^{11,106-109}$. The detection time ranges from several minutes to hours depending on the technology $y^{106,107,109-111}$. The molecular detection can be affected by many factors. Although SARS-CoV-2 has been detected from a variety of respiratory sources, including throat swabs, posterior oropharyngeal saliva, nasopharyngeal swabs, sputum and bronchial fluid, the viral load is higher in lower respiratory tract samples $^{11,96,112-115}$. In addition, viral nucleic acid was also found in samples from the intestinal tract or blood even when respiratory samples were negative ${ }^{116}$. Lastly, viral load may already drop from its peak level on disease onset $^{96,97}$. Accordingly, false negatives can be common when oral swabs and used, and so multiple detection methods should be adopted to confirm a COVID-19 diagnosis ${ }^{117,118}$. Other detection methods were therefore used to overcome this problem. Chest CT was used to quickly identify a patient when the capacity of molecular detection was overloaded in Wuhan. Patients 
with COVID-19 showed typical features on initial CT, including bilateral multilobar ground-glass opacities with a peripheral or posterior distribution ${ }^{118,119}$. Thus, it has been suggested that CT scanning combined with repeated swab tests should be used for individuals with high clinical suspicion of COVID-19 but who test negative in initial nucleic acid screening ${ }^{118}$. Finally, SARS-CoV-2 serological tests detecting antibodies to $\mathrm{N}$ or $\mathrm{S}$ protein could complement molecular diagnosis, particularly in late phases after disease onset or for retrospective studies ${ }^{116,120,121}$. However, the extent and duration of immune responses are still unclear, and available serological tests differ in their sensitivity and specificity, all of which need to be taken into account when one is deciding on serological tests and interpreting their results or potentially in the future test for $\mathrm{T}$ cell responses.

\section{Therapeutics}

To date, there are no generally proven effective therapies for COVID-19 or antivirals against SARS-CoV-2, although some treatments have shown some benefits in certain subpopulations of patients or for certain end points (see later). Researchers and manufacturers are conducting large-scale clinical trials to evaluate various therapies for COVID-19. As of 2 October 2020, there were about 405 therapeutic drugs in development for COVID-19, and nearly 318 in human clinical trials (COVID-19 vaccine and therapeutics tracker). In the following sections, we summarize potential therapeutics against SARS-CoV-2 on the basis of published clinical data and experience.

Inhibition of virus entry. SARS-CoV-2 uses ACE 2 as the receptor and human proteases as entry activators; subsequently it fuses the viral membrane with the cell membrane and achieves invasion. Thus, drugs that interfere with entry may be a potential treatment for COVID-19. Umifenovir (Arbidol) is a drug approved in Russia and China for the treatment of influenza and other respiratory viral infections. It can target the interaction between the $S$ protein and ACE2 and inhibit membrane fusion (FIG. 5). In vitro experiments showed that it has activity against SARS-CoV-2, and current clinical data revealed it may be more effective than lopinavir and ritonavir in treating COVID-19 (REFS ${ }^{122,123}$ ). However, other clinical studies showed umifenovir might not improve the prognosis of or accelerate SARS-CoV-2 clearance in patients with mild to moderate COVID-19 (REFS ${ }^{124,125}$ ). Yet some ongoing clinical trials are evaluating its efficacy for COVID-19 treatment. Camostat mesylate is approved in Japan for the treatment of pancreatitis and postoperative reflux oesophagitis. Previous studies showed that it can prevent SARS-CoV from entering cells by blocking TMPRSS2 activity and protect mice from lethal infection with SARS-CoV in a pathogenic mouse model (wildtype mice infected with a mouse-adapted SARS-CoV strain) $)^{126,127}$. Recently, a study revealed that camostat mesylate blocks the entry of SARS-CoV-2 into human lung cells ${ }^{47}$. Thus, it can be a potential antiviral drug against SARS-CoV-2 infection, although so far there are not sufficient clinical data to support its efficacy.
Chloroquine and hydroxychloroquine are other potential but controversial drugs that interfere with the entry of SARS-CoV-2. They have been used in the prevention and treatment of malaria and autoimmune diseases, including systemic lupus erythematosus and rheumatoid arthritis. They can inhibit the glycosylation of cellular receptors and interfere with virus-host receptor binding, as well as increase the endosomal $\mathrm{pH}$ and inhibit membrane fusion. Currently, no scientific consensus has been reached for their efficacy in the treatment of COVID-19. Some studies showed they can inhibit SARS-CoV-2 infection in vitro, but the clinical data are insufficient ${ }^{128,129}$. Two clinical studies indicated no association with death rates in patients receiving chloroquine or hydroxychloroquine compared with those not receiving the drug and even suggest it may increase the risk of dying as a higher risk of cardiac arrest was found in the treated patients ${ }^{130,131}$. On 15 June 2020, owing to the side effects observed in clinical trials, the US Food and Drug Administration (FDA) revoked the emergency use authorization for chloroquine and hydroxychloroquine for the treatment of COVID-19. Another potential therapeutic strategy is to block binding of the S protein to ACE2 through soluble recombinant hACE2, specific monoclonal antibodies or fusion inhibitors that target the SARS-CoV-2 S protein ${ }^{132-134}$ (FIG. 5). The safety and efficacy of these strategies need to be assessed in future clinical trials.

Inhibition of virus replication. Replication inhibitors include remdesivir (GS-5734), favilavir (T-705), ribavirin, lopinavir and ritonavir. Except for lopinavir and ritonavir, which inhibit 3CLpro, the other three all target $\mathrm{RdRp}^{128,135}$ (FIG. 5). Remdesivir has shown activity against SARS-CoV-2 in vitro and in vivo ${ }^{128,136}$. A clinical study revealed a lower need for oxygen support in patients with COVID-19 (REF. ${ }^{137}$ ). Preliminary results of the Adaptive COVID-19 Treatment Trial (ACTT) clinical trial by the National Institute of Allergy and Infectious Diseases (NIAID) reported that remdesivir can shorten the recovery time in hospitalized adults with COVID-19 by a couple days compared with placebo, but the difference in mortality was not statistically significant ${ }^{138}$. The FDA has issued an emergency use authorization for remdesivir for the treatment of hospitalized patients with severe COVID-19. It is also the first approved option by the European Union for treatment of adults and adolescents with pneumonia requiring supplemental oxygen. Several international phase III clinical trials are continuing to evaluate the safety and efficacy of remdesivir for the treatment of COVID-19.

Favilavir (T-705), which is an antiviral drug developed in Japan to treat influenza, has been approved in China, Russia and India for the treatment of COVID-19. A clinical study in China showed that favilavir significantly reduced the signs of improved disease signs on chest imaging and shortened the time to viral clearance $^{139}$. A preliminary report in Japan showed rates of clinical improvement of $73.8 \%$ and $87.8 \%$ from the start of favilavir therapy in patients with mild COVID-19 at 7 and 14 days, respectively, and $40.1 \%$ and $60.3 \%$ in patients with severe COVID-19 at 7 and 14 days, 


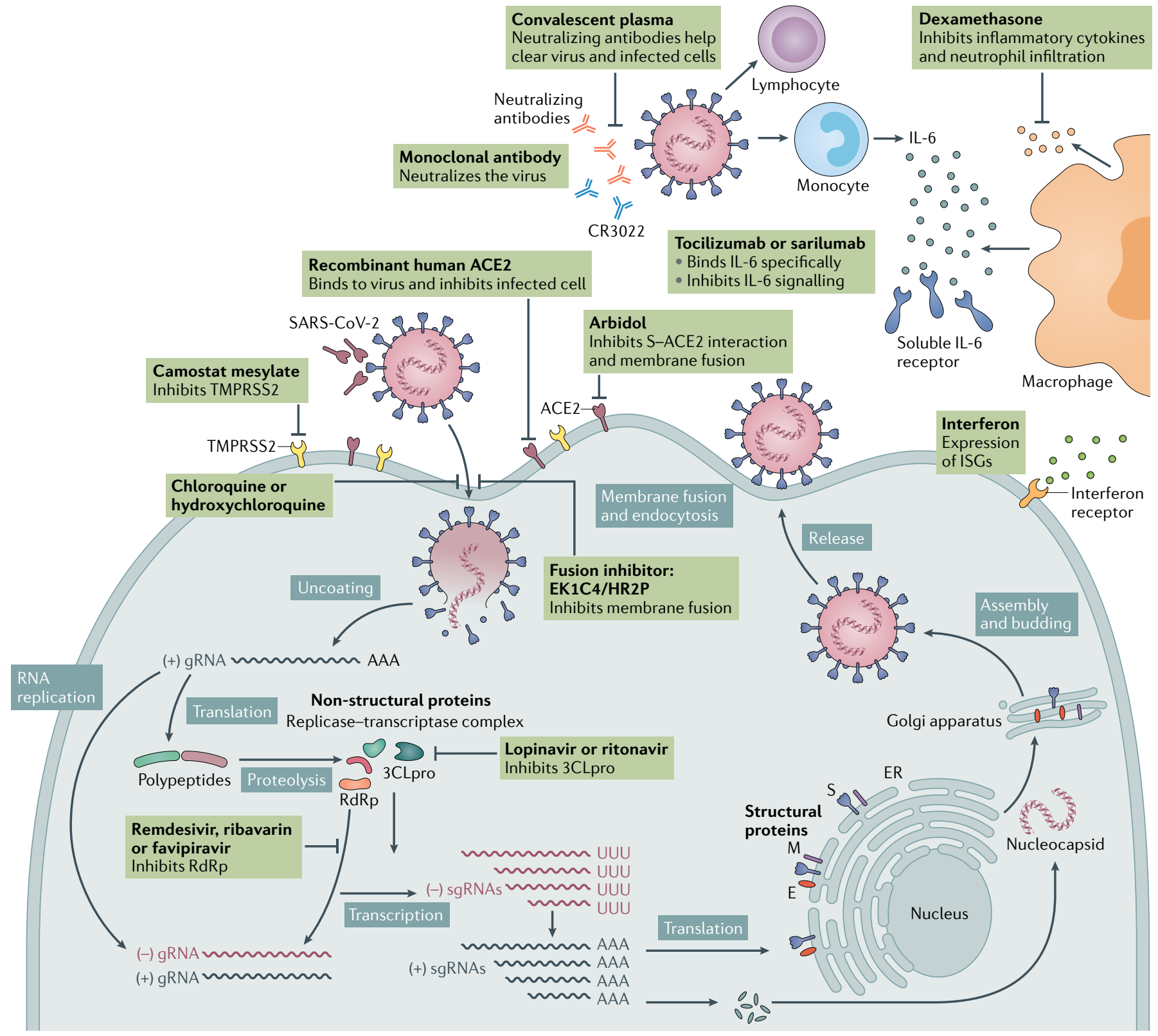

Fig. 5 | SARS-CoV-2 replication and potential therapeutic targets. Potential antivirals target the different steps of severe acute respiratory syndrome coronavirus 2 (SARS-CoV-2) replication, ranging from receptor binding, entry and fusion to replication. Furthermore, immunoglobulin-based and immunomodulatory drugs are potential therapeutics as well. Note that robust data on clinical efficacy are lacking for most of these treatments so far. 3CLpro, 3C-like protease; ACE2, angiotensin-converting enzyme 2; CR3022, a SARS-CoV-specific human monoclonal antibody; E, envelope protein; EK1C4, lipopeptide derived from EK1 which is a pan-coronavirus fusion inhibitor targeting the HR1 domain of the spike protein; ER, endoplasmic reticulum; gRNA, genomic RNA; HR2P, heptad repeat 2-derived peptides of SARS-CoV-2 spike protein; IL-6, interleukin-6; ISG, interferon-stimulated gene; M, membrane protein; RdRp, RNA-dependent RNA polymerases; sgRNA, subgenomic RNA; S, spike protein; TMPRSS2, transmembrane protease serine protease 2.

respectively ${ }^{140}$. However, this study did not include a control arm, and most of the trials of favilavir were based on a small sample size. For more reliable assessment of the effectiveness of favilavir for treating COVID-19, large-scale randomized controlled trials should be conducted.

Lopinavir and ritonavir were reported to have in vitro inhibitory activity against SARS-CoV and MERS-CoV ${ }^{141,142}$. Alone, the combination of lopinavir and ritonavir had little therapeutic benefit in patients with COVID-19, but appeared more effective when used in combination with other drugs, including ribavirin and interferon beta- $1 b^{143,144}$. The Randomized Evaluation of COVID-19 Therapy (RECOVERY) trial, a national clinical trial programme in the UK, has stopped treatment with lopinavir and ritonavir as no significant beneficial effect was observed in a randomized trial established in March 2020 with a total of 1,596 patients ${ }^{145}$. Nevertheless, 
other clinical trials in different phases are still ongoing elsewhere.

Immunomodulatory agents. SARS-CoV-2 triggers a strong immune response which may cause cytokine storm syndrome $e^{60,61}$. Thus, immunomodulatory agents that inhibit the excessive inflammatory response may be a potential adjunctive therapy for COVID-19. Dexamethasone is a corticosteroid often used in a wide range of conditions to relieve inflammation through its anti-inflammatory and immunosuppressant effects. Recently, the RECOVERY trial found dexamethasone reduced mortality by about one third in hospitalized patients with COVID-19 who received invasive mechanical ventilation and by one fifth in patients receiving oxygen. By contrast, no benefit was found in patients without respiratory support ${ }^{146}$.

Tocilizumab and sarilumab, two types of interleukin-6 (IL-6) receptor-specific antibodies previously used to treat various types of arthritis, including rheumatoid arthritis, and cytokine release syndrome, showed effectiveness in the treatment of severe COVID-19 by attenuating the cytokine storm in a small uncontrolled trial ${ }^{147}$. Bevacizumab is an anti-vascular endothelial growth factor (VEGF) medication that could potentially reduce pulmonary oedema in patients with severe COVID-19. Eculizumab is a specific monoclonal antibody that inhibits the proinflammatory complement protein C5. Preliminary results showed that it induced a drop of inflammatory markers and C-reactive protein levels, suggesting its potential to be an option for the treatment of severe COVID-19 (REF. ${ }^{148}$ ).

The interferon response is one of the major innate immunity defences against virus invasion. Interferons induce the expression of diverse interferon-stimulated genes, which can interfere with every step of virus replication. Previous studies identified type I interferons as a promising therapeutic candidate for SARS ${ }^{149}$. In vitro data showed SARS-CoV-2 is even more sensitive to type I interferons than SARS-CoV, suggesting the potential effectiveness of type I interferons in the early treatment of COVID-19 (REF. ${ }^{150}$ ). In China, vapor inhalation of interferon- $\alpha$ is included in the COVID-19 treatment guideline ${ }^{151}$. Clinical trials are ongoing across the world to evaluate the efficacy of different therapies involving interferons, either alone or in combination with other agents ${ }^{152}$.

Immunoglobulin therapy. Convalescent plasma treatment is another potential adjunctive therapy for COVID-19. Preliminary findings have suggested improved clinical status after the treatment ${ }^{153,154}$. The FDA has provided guidance for the use of COVID-19 convalescent plasma under an emergency investigational new drug application. However, this treatment may have adverse effects by causing antibody-mediated enhancement of infection, transfusion-associated acute lung injury and allergic transfusion reactions.

Monoclonal antibody therapy is an effective immunotherapy for the treatment of some viral infections in select patients. Recent studies reported specific monoclonal antibodies neutralizing SARS-CoV-2 infection in vitro and in vivo ${ }^{155-158}$. Compared with convalescent plasma, which has limited availability and cannot be amplified, monoclonal antibodies can be developed in larger quantities to meet clinical requirements. Hence, they provide the possibility for the treatment and prevention of COVID-19. The neutralizing epitopes of these monoclonal antibodies also offer important information for vaccine design. However, the high cost and limited capacity of manufacturing, as well as the problem of bioavailability, may restrict the wide application of monoclonal antibody therapy.

\section{Vaccines}

Vaccination is the most effective method for a long-term strategy for prevention and control of COVID-19 in the future. Many different vaccine platforms against SARS-CoV-2 are in development, the strategies of which include recombinant vectors, DNA, mRNA in lipid nanoparticles, inactivated viruses, live attenuated viruses and protein subunits ${ }^{159-161}$. As of 2 October 2020, 174 vaccine candidates for COVID-19 had been reported and 51 were in human clinical trials (COVID-19 vaccine and therapeutics tracker). Many of these vaccine candidates are in phase II testing, and some have already advanced to phase III trials. A randomized double-blinded phase II trial of an adenovirus type 5vectored vaccine expressing the SARS-CoV-2 S protein, developed by CanSino Biologicals and the Academy of Military Medical Sciences of China, was conducted in 603 adult volunteers in Wuhan. The vaccine has proved to be safe and induced considerable humoral and cellular immune response in most recipients after a single immunization $^{162}$. Another vectored vaccine, ChAdOx1, was developed on the basis of chimpanzee adenovirus by the University of Oxford. In a randomized controlled phase I/II trial, it induced neutralizing antibodies against SARS-CoV-2 in all 1,077 participants after a second vaccine dose, while its safety profile was acceptable as well ${ }^{163}$. The NIAID and Moderna co-manufactured mRNA-1273, a lipid nanoparticle-formulated mRNA vaccine candidate that encodes the stabilized prefusion SARS-CoV-2 S protein. Its immunogenicity has been confirmed by a phase I trial in which robust neutralizing antibody responses were induced in a dose-dependent manner and increased after a second dose ${ }^{164}$. Regarding inactivated vaccines, a successful phase I/II trial involving 320 participants has been reported in China. The whole-virus COVID-19 vaccine had a low rate of adverse reactions and effectively induced neutralizing antibody production ${ }^{165}$. The verified safety and immunogenicity support advancement of these vaccine candidates to phase III clinical trials, which will evaluate their efficacy in protecting healthy populations from SARS-CoV-2 infection.

\section{Future perspectives}

COVID-19 is the third highly pathogenic human coronavirus disease to date. Although less deadly than SARS and MERS, the rapid spreading of this highly contagious disease has posed the severest threat to global health in this century. The SARS-CoV-2 outbreak has lasted for more than half a year now, and it is likely that 
this emerging virus will establish a niche in humans and coexist with us for a long time ${ }^{166}$. Before clinically approved vaccines are widely available, there is no better way to protect us from SARS-CoV-2 than personal preventive behaviours such as social distancing and wearing masks, and public health measures, including active testing, case tracing and restrictions on social gatherings. Despite a flood of SARS-CoV-2 research published every week, current knowledge of this novel coronavirus is just the tip of the iceberg. The animal origin and cross-species infection route of SARS-CoV-2 are yet to be uncovered. The molecular mechanisms of SARS-CoV-2 infection pathogenesis and virus-host interactions remain largely unclear. Intensive studies on these virological profiles of SARS-CoV-2 will provide the basis for the development of preventive and therapeutic strategies against COVID-19. Moreover, continued genomic monitoring of SARS-CoV-2 in new cases is needed worldwide, as it is important to promptly identify any mutation that may result in phenotypic changes of the virus. Finally, COVID-19 is challenging all human beings. Tackling this epidemic is a long-term job which requires efforts of every individual, and international collaborations by scientists, authorities and the public.

Published online 6 October 2020
1. Cui, J., Li, F. \& Shi, Z. L. Origin and evolution of pathogenic coronaviruses. Nat. Rev. Microbiol. 17, 181-192 (2019)

2. Wu, J. T., Leung, K. \& Leung, G. M. Nowcasting and forecasting the potential domestic and international spread of the 2019-nCoV outbreak originating in Wuhan, China: a modelling study. Lancet 395, 689-697 (2020).

3. Hui, D. S. et al. The continuing 2019-nCoV epidemic threat of novel coronaviruses to global health - the latest 2019 novel coronavirus outbreak in Wuhan China. Intl. J. Infect. Dis. 91, 264-266 (2020).

4. Deng, S. Q. \& Peng, H. J. Characteristics of and public health responses to the coronavirus disease 2019 outbreak in China. J. Clin. Med. 9, 575 (2020).

5. Han, Q., Lin, Q., Jin, S. \& You, L. Coronavirus 2019 nCoV: a brief perspective from the front line. J. Infect. 80, 373-377 (2020)

6. Zhu, N. et al. A Novel Coronavirus from patients with pneumonia in China, 2019. N. Engl. J. Med. 382 727-733 (2020)

7. Gralinski, L. E. \& Menachery, V. D. Return of the coronavirus: 2019-nCoV. Viruses 12, 135 (2020).

8. Jiang, S., Du, L. \& Shi, Z. An emerging coronavirus causing pneumonia outbreak in Wuhan, China: calling for developing therapeutic and prophylactic strategies. Emerg. Microbes Infect. 9, 275-277 (2020).

9. Wu, Z. \& McGoogan, J. M. Characteristics of and important lessons from the coronavirus disease 2019 (COVID-19) outbreak in china: summary of a report of 72314 cases from the Chinese Center for Disease Control and Prevention. JAMA 323, 1239-1242 (2020).

10. Wu, F. et al. A new coronavirus associated with human respiratory disease in China. Nature 579, 265-269 (2020).

11. Zhou, P. et al. A pneumonia outbreak associated with a new coronavirus of probable bat origin. Nature $\mathbf{5 7 9}$ 270-273 (2020).

12. Chan, J. F. et al. A familial cluster of pneumonia associated with the 2019 novel coronavirus indicating person-to-person transmission: a study of a family cluster. Lancet 395, 514-523 (2020)

13. Chen, N. et al. Epidemiological and clinical characteristics of 99 cases of 2019 novel coronavirus pneumonia in Wuhan, China: a descriptive study. Lancet 395, 507-513 (2020).

14. Wang, R., Zhang, X., Irwin, D. M. \& Shen, Y Emergence of SARS-like coronavirus poses new challenge in China. J. Infect. 80, 350-371 (2020).

15. National Health Commission of the People's Republic of China. Briefing on the latest situation of the novel coronavirus pneumonia epidemic http://www.nhc.gov.cn/xcs/yqtb/list_gzbd.shtml (2020).

16. Eurosurveillance Editorial Team. Note from the editors: World Health Organization declares novel coronavirus (2019-nCoV) sixth public health emergency of international concern. Euro. Surveill. 25, 200131e (2020).

17. Coronaviridae Study Group of the International Committee on Taxonomy of Viruses. The species severe acute respiratory syndrome-related coronavirus: classifying 2019-nCoV and naming it SARS-CoV-2. Nat. Microbiol. 5, 536-544 (2020)

18. Fisher, D. \& Heymann, D. O\&A: the novel coronavirus outbreak causing COVID-19. BMC Med. 18, 57 (2020).

19. Lai, C. C., Shih, T. P., Ko, W. C., Tang, H. J. $\&$ Hsueh, P. R. Severe acute respiratory syndrome coronavirus 2 (SARS-CoV-2) and coronavirus disease-2019 (COVID-19): the epidemic and the challenges. Int. J. Antimicrob. Agents 55, 105924 (2020).

20. World Health Organization. Coronavirus disease 2019 (COVID-19). Situation report - 51. https:// www.who.int/docs/default-source/coronaviruse/ situation-reports/20200311-sitrep-51-covid-19. pdf?sfvrsn=1 ba62e57_10 (2020).

21. Dong, E., Du, H. \& Gardner, L. An interactive web-based dashboard to track COVID-19 in real time. Lancet Infect. Dis. 20, 533-534 (2020).

22. Li, Q. et al. Early transmission dynamics in Wuhan, China, of novel coronavirus-infected pneumonia N. Engl. J. Med. 382, 1199-1207 (2020).

23. Deslandes, A. et al. SARS-CoV-2 was already spreading in France in late December 2019. Int. J. Antimicrob. Agents 55, 106006 (2020).

24. Lu, R. et al. Genomic characterisation and epidemiology of 2019 novel coronavirus: implications for virus origins and receptor binding. Lancet 395, 565-574 (2020).

25. Chan, J. F. et al. Genomic characterization of the 2019 novel human-pathogenic coronavirus isolated from a patient with atypical pneumonia after visiting Wuhan. Emerg. Microbes Infect. 9, 221-236 (2020).

26. Anderson, K. G., Rambaut, A., Lipkin, W. I., Holmes, E. C. \& Garry, R. F. The proximal origin of SARS-CoV-2. Nat. Med. 26, 450-452 (2020).

27. Coutard, B. et al. The spike glycoprotein of the new coronavirus 2019-nCoV contains a furin-like cleavage site absent in CoV of the same clade. Antiviral Res. 176, 104742 (2020)

28. Zhou, H. et al. A novel bat coronavirus closely related to SARS-CoV-2 contains natural insertions at the S1/S2 cleavage site of the spike protein. Curr. Biol. 30 2196-2203 (2020).

29. Wrobel, A. G. et al. SARS-CoV-2 and bat RaTG 13 spike glycoprotein structures inform on virus evolution and furin-cleavage effects. Nat. Struct. Mol. Biol. 27, 763-767 (2020)

30. Su, Y. C. F. et al. Discovery and genomic characterization of a 382-nucleotide deletion in ORF7b and ORF8 during the early evolution of SARS-CoV-2. mBio 11 e01610-20 (2020)

31. Zhao, W. M. et al. The 2019 novel coronavirus resource. Yi Chuan 42, 212-221 (2020).

32. Korber, B. et al. Tracking changes in SARS-CoV-2 spike: evidence that D614G increases infectivity of the COVID-19 virus. Cell 182, 812-827 (2020)

33. Tang, $X$. et al. On the origin and continuing evolution of SARS-CoV-2. Natl Sci. Rev. 7, 1012-1023 (2020).

34. Forster, P., Forster, L., Renfrew, C. \& Forster, M. Phylogenetic network analysis of SARS-CoV-2 genomes. Proc. Natl Acad. Sci. USA 117, 9241-9243 (2020).

35. Paraskevis, D. et al. Full-genome evolutionary analysis of the novel corona virus (2019-nCoV) rejects the hypothesis of emergence as a result of a recent recombination event. Infect. Genet. Evol. 79, 104212 (2020).

36. Hu, D. et al. Genomic characterization and infectivity of a novel SARS-like coronavirus in Chinese bats. Emerg. Microbes Infect. 7, 154 (2018).

37. Lau, S. K. P. et al. Possible bat origin of severe acute respiratory syndrome coronavirus 2 . Emerg. Infect. Dis. 26, 1542-1547 (2020)

38. Zhang, Y. Z. \& Holmes, E. C. A genomic perspective on the origin and emergence of SARS-CoV-2. Cell 181 223-227 (2020)

39. Lam, T. T. et al. Identifying SARS-CoV-2 related coronaviruses in Malayan pangolins. Nature $\mathbf{5 8 3}$ 282-285 (2020).
40. Xiao $\mathrm{K}$ et al Isolation of SARS-CoV-2-related coronavirus from Malayan pangolins. Nature $\mathbf{5 8 3}$, 286-289 (2020).

41. Liu, P., Chen, W. \& Chen, J. P. Viral metagenomics revealed Sendai virus and coronavirus infection of Malayan pangolins (Manis javanica). Viruses 11, 979 (2019).

42. Zhang, T., Wu, Q. \& Zhang, Z. Probable pangolin origin of SARS-CoV-2 associated with the COVID-19 outbreak. Curr Biol 30, 1346-1351 (2020).

43. Shi, J. et al. Susceptibility of ferrets, cats, dogs, and other domesticated animals to SARS-coronavirus 2. Science 368, 1016-1020 (2020).

44. Oreshkova, N. et al. SARS-CoV-2 infection in farmed minks, the Netherlands, April and May 2020. Euro Surveill. 25, 2001005 (2020)

45. Sit, T. H. C. et al. Infection of dogs with SARS-CoV-2. Nature https://doi.org/10.1038/s41586-020-2334-5 (2020).

46. Zhang, $\mathrm{Q}$. et al. A serological survey of SARS-CoV-2 in cat in Wuhan. Emerg. Microbes Infect. https://doi.org/ 10.1080/22221751.2020.1817796 (2020).

47. Hoffmann, M. et al. SARS-CoV-2 cell entry depends on ACE2 and TMPRSS2 and is blocked by a clinically proven protease inhibitor. Cell 181, 271-280 (2020).

48. Chandrashekar, A. et al. SARS-CoV-2 infection protects against rechallenge in rhesus macaques. Science 369 , 812-817 (2020)

49. Zhao, X. et al. Broad and differential animal angiotensin-converting enzyme 2 receptor usage by SARS-CoV-2. J. Virol. 94, e00940-20 (2020).

50. Shang, J. et al. Structural basis of receptor recognition by SARS-CoV-2. Nature 581, 221-224 (2020).

51. Walls, A. C. et al. Structure, function, and antigenicity of the SARS-CoV-2 spike glycoprotein. Cell 181 , 281-292 (2020).

52. Wan, Y., Shang, J., Graham, R., Baric, R. S. \& Li, F. Receptor recognition by the novel coronavirus from Wuhan: an analysis based on decade-long structural studies of SARS coronavirus. J. Virol. 94, e00127-20 (2020).

53. Letko, M., Marzi, A. \& Munster, V. Functional assessment of cell entry and receptor usage for SARS CoV-2 and other lineage B betacoronaviruses. Nat. Microbiol. 5, 562-569 (2020).

54. Ou, X. et al. Characterization of spike glycoprotein of SARS-CoV- 2 on virus entry and its immune crossreactivity with SARS-CoV. Nat. Commun. 11, 1620 (2020).

55. Shang, J. et al. Cell entry mechanisms of SARS-CoV-2 Proc. Natl Acad. Sci. USA 117, 11727-11734 (2020).

56. Sungnak, W. et al. SARS-CoV-2 entry factors are highly expressed in nasal epithelial cells together with innate immune genes. Nat. Med. 26, 681-687 (2020).

57. Lukassen, S. et al. SARS-CoV-2 receptor ACE2 and TMPRSS2 are primarily expressed in bronchial transient secretory cells. EMBO. J. 39, e 105114 (2020).

58. Wrapp, D. et al. Cryo-EM structure of the 2019-nCoV spike in the prefusion conformation. Science 367 1260-1263 (2020).

59. Yuan, Y. et al. Cryo-EM structures of MERS-CoV and SARS-CoV spike glycoproteins reveal the dynamic receptor binding domains. Nat Commun 8, 15092 (2017).

60. Huang, C. et al. Clinical features of patients infected with 2019 novel coronavirus in Wuhan, China. Lancet 395, 497-506 (2020) 
61. Mehta, P. et al. COVID-19: consider cytokine storm syndromes and immunosuppression. Lancet 395 1033-1034 (2020).

62. Wu, C. et al. Risk factors associated with acute respiratory distress syndrome and death in patients with Coronavirus disease 2019 pneumonia in Wuhan, China. JAMA Intern. Med. 180, 934-943 (2020)

63. Liu, Y. et al. Association between age and clinical characteristics and outcomes of COVID-19. Eur. Respir. J. 55, 2001112 (2020).

64. Tian, J. et al. Clinical characteristics and risk factors associated with COVID-19 disease severity in patients with cancer in Wuhan, China: a multicentre, retrospective, cohort study. Lancet Oncol. 21, 893-903 (2020)

65. Yao, X. H. et al. [A pathological report of three COVID-19 cases by minimal invasive autopsies] Zhonghua Bing Li Xue Za Zhi 49, 411-417 (2020).

66. Martines, R. B. et al. Pathology and pathogenesis of SARS-CoV-2 associated with fatal coronavirus disease, United States. Emerg. Infect. Dis. 26, 2005-2015 (2020).

67. Zeng, Z. et al. Pulmonary pathology of early phase COVID-19 pneumonia in a patient with a Benign lung lesion. Histopathology https://doi.org/10.1111/ his. 14138 (2020).

68. Sun, S. H. et al. A mouse model of SARS-CoV-2 infection and pathogenesis. Cell Host Microbe 28 124-133 (2020)

69. Rockx, B. et al. Comparative pathogenesis of COVID-19, MERS, and SARS in a nonhuman primate model. Science 368, 1012-1015 (2020).

70. Bao, L. et al. The pathogenicity of SARS-CoV-2 in hACE2 transgenic mice. Nature 583, 830-833 (2020).

71. Jiang, R. D. et al. Pathogenesis of SARS-CoV-2 in transgenic mice expressing human angiotensinconverting enzyme 2. Cell 182, 50-58 (2020).

72. Lu, S. et al. Comparison of nonhuman primates identified the suitable model for COVID-19. Sig. Transduct. Target. Ther. 5, 157 (2020).

73. Speranza, E. et al. SARS-CoV-2 infection dynamics in lungs of African green monkeys. Preprint at biorxiv https://www. biorxiv.org/content/10.1101/2020.08. 20.258087v1 (2020)

74. Gu, H. et al. Adaptation of SARS-CoV-2 in BALB/c mice for testing vaccine efficacy. Science https://doi.org 10.1126/science.abc4730 (2020)

75. Munster, V. J. et al. Respiratory disease in rhesus macaques inoculated with SARS-CoV-2. Nature 585 268-272 (2020)

76. Yu, P. et al. Age-related rhesus macaque models of COVID-19. Animal Model Exp. Med. 3, 93-97 (2020).

77. Chan, J. F. et al. Simulation of the clinical and pathological manifestations of coronavirus disease 2019 (COVID-19) in golden Syrian hamster model: implications for disease pathogenesis and transmissibility. Clin. Infect. Dis. https://doi.org/10.1093/ $\mathrm{cid} / \mathrm{ciaa325}$ (2020)

78. Kim, Y. I. et al. Infection and rapid transmission of SARS-CoV-2 in ferrets. Cell Host Microbe 27 704-709 (2020).

79. Richard, M. et al. SARS-CoV-2 is transmitted via contact and via the air between ferrets. Nat. Commun. 11, 3496 (2020).

80. Wang, D. et al. Clinical characteristics of 138 hospitalized patients with 2019 novel coronavirusinfected pneumonia in Wuhan, China. JAMA 323 1061-1069 (2020)

81. Guan, W. J. et al. Clinical characteristics of coronavirus disease 2019 in China. N. Engl. J. Med. 382 1708-1720 (2020).

82. Lu, X. et al. SARS-CoV-2 infection in children. N. Engl. J. Med. 382, 1663-1665 (2020)

83. Chen, $\mathrm{H}$. et al. Clinical characteristics and intrauterine vertical transmission potential of COVID-19 infection in nine pregnant women: a retrospective review of medical records Lancet 395, 809-815 (2020).

84. Vivanti, A. J. et al. Transplacental transmission of SARS CoV-2 infection. Nat. Commun. 11, 3572 (2020).

85. Giacomelli, A. et al. Self-reported olfactory and taste disorders in SARS-CoV-2 patients: a cross-sectional study. Clin. Infect. Dis. 71, 889-890 (2020).

86. Chen, T. et al. Clinical characteristics of 113 deceased patients with coronavirus disease 2019: retrospective study. BMJ 368, m1091 (2020).

87. Yang, X. et al. Clinical course and outcomes of critically ill patients with SARS-CoV-2 pneumonia in Wuhan, China: a single-centered, retrospective, observational study. Lancet Respir. Med. 8, 475-481 (2020).

88. Nishiura, H., Linton, N. M. \& Akhmetzhanov, A. R. Initial cluster of novel coronavirus (2019-nCoV) infections in Wuhan, China is consistent with substantial human-to-human transmission. J. Clin. Med. 9, 488 (2020).

89. Li, R. et al. Substantial undocumented infection facilitates the rapid dissemination of novel coronavirus (SARS-CoV-2). Science 368, 489-493 (2020).

90. Petersen, E. et al. Comparing SARS-CoV-2 with SARS$\mathrm{CoV}$ and influenza pandemics. Lancet Infect. Dis. 20, e238-e244 (2020)

91. Yu, P., Zhu, J., Zhang, Z. \& Han, Y. A familial cluster of infection associated with the 2019 novel coronavirus indicating possible person-to-person transmission during the incubation period. J. Infect. Dis. 221, 1757-1761 (2020).

92. Middleton, J., Reintjes, R. \& Lopes, H. Meat plants-a new front line in the covid-19 pandemic. BMJ 370 . $\mathrm{m} 2716$ (2020)

93. Joob, B. \& Wiwanitkit, V. COVID-19 and migrant workers: lack of data and need for specific management. Public Health 183, 64 (2020).

94. Houlihan, C. F. et al. Pandemic peak SARS-CoV-2 infection and seroconversion rates in London frontline health-care workers. Lancet 396, e6-e7 (2020).

95. Peiris, J. S. et al. Clinical progression and viral load in a community outbreak of coronavirus-associated SARS pneumonia: a prospective study. Lancet 361 , 1767-1772 (2003).

96. Zou, L. et al. SARS-CoV-2 viral load in upper respiratory specimens of infected patients. N. Engl. J. Med. 382, 1177-1179 (2020).

97. Wolfel, R. et al. Virological assessment of hospitalized patients with COVID-2019. Nature https://doi.org/ 10.1038/s41586-020-2196-x (2020)

98. Stadnytskyi, V., Bax, C. E., Bax, A. \& Anfinrud, P. The airborne lifetime of small speech droplets and their potential importance in SARS-CoV-2 transmission. Proc. Natl Acad. Sci. USA 117, 11875-11877 (2020).

99. Meselson, M. Droplets and aerosols in the transmission of SARS-CoV-2. N. Engl. J. Med. 382 , 2063 (2020)

100. van Doremalen, N. et al. Aerosol and surface stability of SARS-CoV-2 as compared with SARS-CoV-1. N. Engl. J. Med. 382, 1564-1567 (2020).

101. Lu, C. W., Liu, X. F. \& Jia, Z. F. 2019-nCoV transmission through the ocular surface must not be ignored. Lancet 395, e39 (2020).

102. Wu, Y. et al. Prolonged presence of SARS-CoV-2 viral RNA in faecal samples. Lancet Gastroenterol. Hepatol. 5, 434-435 (2020).

103. Kampf, G., Todt, D., Pfaender, S. \& Steinmann, E. Persistence of coronaviruses on inanimate surfaces and their inactivation with biocidal agents. J. Hosp. Infect. 104, 246-251 (2020).

104. Chinazzi, M. et al. The effect of travel restrictions on the spread of the 2019 novel coronavirus (COVID-19) outbreak. Science 368, 395-400 (2020).

105. Lu, N., Cheng, K. W., Qamar, N., Huang, K. C. \& Johnson, J. A. Weathering COVID-19 storm: successful control measures of five Asian countries. Am. J. Infect. Control 48, 851-852 (2020).

106. Bordi, L. et al. Differential diagnosis of illness in patients under investigation for the novel coronavirus (SARS-CoV-2), Italy, February 2020. Euro Surveill. 25 , 2000170 (2020)

107. Chan, J. F. et al. Improved molecular diagnosis of COVID-19 by the Novel, highly sensitive and specific COVID-19-RdRp/Hel real-time reverse transcriptionPCR assay validated in vitro and with clinical specimens. J. Clin. Microbiol. 58, e00310-20 (2020).

108. Corman, V. M. et al. Detection of 2019 novel coronavirus (2019-nCoV) by real-time RT-PCR. Euro Surveill. 25, 2000045 (2020).

109. Konrad, R. et al. Rapid establishment of laboratory diagnostics for the novel coronavirus SARS-CoV-2 in Bavaria, Germany, February 2020. Euro Surveill. 25, 2000173 (2020)

110. Lu, R et al. Development of a novel reverse transcription loop-mediated isothermal amplification method for rapid detection of SARS-CoV-2. Virol. Sin. 35, 344-347 (2020).

111. Cordes, A. K. \& Heim, A. Rapid random access detection of the novel SARS-coronavirus-2 (SARS-CoV-2, previously 2019-nCoV) using an open access protocol for the panther fusion. J. Clin. Virol. 125, 104305 (2020).

112. Pan, Y., Zhang, D., Yang, P., Poon, L. L. M. \& Wang, Q. Viral load of SARS-CoV-2 in clinical samples. Lancet Infect. Dis. 20, 411-412 (2020).

113. To, K. K. et al. Consistent detection of 2019 novel coronavirus in saliva. Clin. Infect. Dis. 71, 841-843 (2020).
114. Wang, W. et al. Detection of SARS-CoV-2 in different types of clinical specimens. JAMA 323, 1843-1844 (2020).

115. Han, H., Luo, Q., Mo, F., Long, L. \& Zheng, W. SARS-CoV-2 RNA more readily detected in induced sputum than in throat swabs of convalescent COVID-19 patients. Lancet Infect. Dis. 20, 655-656 (2020).

116. Zhang, W. et al. Molecular and serological investigation of 2019-nCoV infected patients: implication of multiple shedding routes. Emerg. Microbes Infect. 9, 386-389 (2020).

117. Li, T. Diagnosis and clinical management of severe acute respiratory syndrome coronavirus 2 (SARS-CoV-2) infection: an operational recommendation of Peking Union Medical College Hospital (V2.0). Emerg. Microbes Infect. 9, 582-585 (2020).

118. Xie, X. et al. Chest CT for typical 2019-nCoV pneumonia: relationship to negative RT-PCR testing Radiology 296, E41-E45 (2020).

119. Kanne, J. P. \& Chest, C. T. Findings in 2019 novel coronavirus (2019-nCoV) infections from Wuhan, China: key points for the radiologist. Radiology 295, 16-17 (2020)

120. Guo, L. et al. Profiling early humoral response to diagnose novel coronavirus disease (COVID-19). Clin. Infect. Dis. 71, 778-785 (2020).

121. To, K. K. et al. Temporal profiles of viral load in posterior oropharyngeal saliva samples and serum antibody responses during infection by SARS-CoV-2: an observational cohort study. Lancet Infect. Dis. 20 565-574 (2020)

122. Wang, X. et al. The anti-influenza virus drug, arbidol is an efficient inhibitor of SARS-CoV-2 in vitro. Cell Discov. 6, 28 (2020).

123. Zhu, Z. et al. Arbidol monotherapy is superior to lopinavir/ritonavir in treating COVID-19. J. Infect. 81 e21-e23 (2020).

124. Li, Y. et al. Efficacy and safety of lopinavir/ritonavir or arbidol in adult patients with mild/moderate COVID-19: an exploratory randomized controlled trial. Med https://doi.org/10.1016/j.medj.2020.04.001 (2020).

125. Lian, N. et al. Umifenovir treatment is not associated with improved outcomes in patients with coronavirus disease 2019: a retrospective study. Clin. Microbiol. Infect. 26, 917-921 (2020)

126. Kawase, M., Shirato, K. van der Hoek, L., Taguchi, F. \& Matsuyama, S. Simultaneous treatment of human bronchial epithelial cells with serine and cysteine protease inhibitors prevents severe acute respiratory syndrome coronavirus entry. J. Virol. 86, 6537-6545 (2012).

127. Zhou, Y. et al. Protease inhibitors targeting coronavirus and filovirus entry. Antiviral Res. 116, 76-84 (2015).

128. Wang, M. et al. Remdesivir and chloroquine effectively inhibit the recently emerged novel coronavirus (2019nCoV) in vitro. Cell Res. 30, 269-271 (2020).

129. Yao, X. et al. In vitro antiviral activity and projection of optimized dosing design of hydroxychloroquine for the treatment of severe acute respiratory syndrome coronavirus 2 (SARS-CoV-2). Clin. Infect. Dis. 71 , 732-739 (2020)

130. Rosenberg, E. S. et al. Association of treatment with hydroxychloroquine or azithromycin with in-hospital mortality in patients with COVID-19 in New York state. JAMA 323, 2493-2502 (2020).

131. Geleris, J. et al. Observational study of hydroxychloroquine in hospitalized patients with Covid- 19. N. Engl. J. Med. 382, 2411-2418 (2020).

132. Monteil, V. et al. Inhibition of SARS-CoV-2 infections in engineered human tissues using clinical-grade soluble human ACE2. Cell 181, 905-913 (2020).

133. Tian, X. et al. Potent binding of 2019 nove coronavirus spike protein by a SARS coronavirusspecific human monoclonal antibody. Emerg. Microbes Infect. 9, 382-385 (2020).

134. Xia, S. et al. Inhibition of SARS-CoV-2 (previously 2019$\mathrm{nCoV}$ ) infection by a highly potent pan-coronavirus fusion inhibitor targeting its spike protein that harbors a high capacity to mediate membrane fusion. Cell Res. 30, 343-355 (2020)

135. Ul Qamar, M. T., Alqahtani, S. M., Alamri, M. A $\&$ Chen, L. L. Structural basis of SARS-CoV-2 $3 \mathrm{CL}$ (pro) and anti-COVID-19 drug discovery from medicinal plants. J. Pharm. Anal. 10, 313-319 (2020).

136. Williamson, B. N. et al. Clinical benefit of remdesivir in rhesus macaques infected with SARS-CoV-2. Nature 585, 273-276 (2020).

137. Grein, J. et al. Compassionate use of remdesivir for patients with severe Covid-19. N. Engl. J. Med. 382, 2327-2336 (2020). 
138. Beigel, J. H. et al. Remdesivir for the treatment of Covid-19 - preliminary report. N. Engl. J. Med https:// doi.org/10.1056/NEJMoa2007764 (2020).

139. Cai, Q. et al. Experimental treatment with favipiravir for COVID-19: an open-label control study. Engineering https://doi.org/10.1016/i.eng.2020.03.007 (2020).

140. Favipiravir Observational Study Group. Preliminary report of the Favipiravir Observational Study in Japan. The Japanese Association for Infectious Diseases. http://www.kansensho.or.jp/uploads/files/topics/ 2019ncov/covid19_casereport_en_200529.pdf (2020).

141. Chen, F. et al. In vitro susceptibility of 10 clinical isolates of SARS coronavirus to selected antiviral compounds. J. Clin. Virol. 31, 69-75 (2004).

142. de Wilde, A. H. et al. Screening of an FDA-approved compound library identifies four small-molecule inhibitors of Middle East respiratory syndrome coronavirus replication in cell culture. Antimicrob. Agents Chemother. 58, 4875-4884 (2014).

143. Cao, B. et al. A trial of lopinavir-ritonavir in adults hospitalized with severe Covid-19. N. Engl. J. Med. 382, 1787-1799 (2020)

144. Hung, I. F. et al. Triple combination of interferon beta-1 $1 \mathrm{~b}$, lopinavir-ritonavir, and ribavirin in the treatment of patients admitted to hospital with COVID-19: an open-label, randomised, phase 2 trial. Lancet 395, 1695-1704 (2020).

145. Chief Investigators of the RECOVERY Trial on Lopinavir-Ritonavir. No clinical benefit from use of lopinavir-ritonavir in hospitalised COVID-19 patients studied in RECOVERY. Randomised Evaluation of COVID-19 Therapy (RECOVERY) trial. https:// www.recoverytrial.net/news/no-clinical-benefitfrom-use-of-lopinavir-ritonavir-in-hospitalised-covid19-patients-studied-in-recovery (2020).

146. Recovery Collaborative Group. et al. Dexamethason in hospitalized patients with Covid-19 - preliminary report. N. Engl. J. Med. https://doi.org/10.1056/ NEJMoa2021436 (2020).

147. Xu, X. et al. Effective treatment of severe COVID-19 patients with tocilizumab. Proc. Natl Acad. Sci. USA 117, 10970-10975 (2020)

148. Diurno, F. et al. Eculizumab treatment in patients with COVID-19: preliminary results from real life ASL Napoli 2 Nord experience. Eur. Rev. Med. Pharmacol. Sci. 24, 4040-4047 (2020).
149. Stockman, L. J., Bellamy, R. \& Garner, P. SARS: systematic review of treatment effects. PLoS Med. 3 , e343 (2006)

150. Mantlo, E., Bukreyeva, N., Maruyama, J., Paessler, S. \& Huang, C. Antiviral activities of type I interferons to SARS-CoV-2 infection. Antiviral Res. 179, 104811 (2020).

151. Sallard, E., Lescure, F. X., Yazdanpanah, Y., Mentre, F. $\&$ Peiffer-Smadja, N. Type 1 interferons as a potential treatment against COVID-19. Antiviral Res. 178, 104791 (2020).

152. Park, A., Iwasaki, A. \& Type, I. and Type III interferons induction, signaling, evasion, and application to combat COVID-19. Cell Host Microbe 27, 870-878 (2020).

153. Duan, K. et al. Effectiveness of convalescent plasma therapy in severe COVID-19 patients. Proc. Natl Acad. Sci. USA 117, 9490-9496 (2020).

154. Shen, C. et al. Treatment of 5 critically ill patients with COVID-19 with convalescent plasma. JAMA 323 1582-1589 (2020).

155. Wang, C. et al. A human monoclonal antibody blocking SARS-CoV-2 infection. Nat. Commun. 11, 2251 (2020)

156. Wu, Y. et al. A noncompeting pair of human neutralizing antibodies block COVID-19 virus binding to its receptor ACE2. Science 368, 1274-1278 (2020).

157. Zost, S. J. et al. Potently neutralizing and protective human antibodies against SARS-CoV-2. Nature 584 443-449 (2020).

158. Shi, R. et al. A human neutralizing antibody targets the receptor-binding site of SARS-CoV-2. Nature $\mathbf{5 8 4}$, 120-124 (2020)

159. Smith, T. R. F. et al. Immunogenicity of a DNA vaccine candidate for COVID-19. Nat. Commun. 11, 2601 (2020).

160. Zhu, F. C. et al. Safety, tolerability, and immunogenicity of a recombinant adenovirus type- 5 vectored COVID-19 vaccine: a dose-escalation, open-label, non-randomised first-in-human trial. Lancet 395, 1845-1854 (2020).

161. Gao, Q. et al. Development of an inactivated vaccine candidate for SARS-CoV-2. Science 369, 77-81 (2020).

162. Zhu, F. C. et al. Immunogenicity and safety of a recombinant adenovirus type-5-vectored COVID-19 vaccine in healthy adults aged 18 years or older: a randomised, double-blind, placebo-controlled, phase 2 trial. Lancet 396, 479-488 (2020).
163. Folegatti, P. M et al. Safety and immunogenicity of the ChAdOx $1 \mathrm{nCoV}-19$ vaccine against SARS-CoV-2: a preliminary report of a phase $1 / 2$, single-blind, randomised controlled trial. Lancet 396, 467-478 (2020).

164. Jackson, L. A. et al. An mRNA vaccine against SARSCoV-2 - preliminary report. N. Engl. J. Med. https:// doi.org/10.1056/NEJMoa2022483 (2020).

165. Xia, S. et al. Effect of an inactivated vaccine against SARS-CoV-2 on safety and immunogenicity outcomes: interim analysis of 2 randomized clinical trials. JAMA 324, 1-10 (2020)

166. Tang, D., Comish, P. \& Kang, R. The hallmarks of COVID-19 disease. PLoS Pathog. 16, e1008536 (2020).

\section{Acknowledgements}

This work was supported by the Strategic Priority Research Program of the Chinese Academy of Sciences (XDB29010101) and the National Natural Science Foundation of China (31770175).

\section{Author contributions}

H.G. and B.H researched data for the article. Z.-L.S. contributed substantially to discussion of the content. H.G., B.H. and P.Z. wrote the article. Z.-L.S. reviewed and edited the manuscript before submission.

\section{Competing interests}

The authors declare no competing interests.

\section{Peer review information}

Nature Reviews Microbiology thanks Vincent Munster and the other, anonymous, reviewer(s) for their contribution to the peer review of this work.

\section{Publisher's note}

Springer Nature remains neutral with regard to jurisdictional claims in published maps and institutional affiliations.

\section{RELATED LINKS}

BIGD: https://bigd.big.ac.cn/ncov/variation/

COVID-19 vaccine and therapeutics tracker

https://biorender.com/covid-vaccine-tracker/

(c) Springer Nature Limited 2020, corrected publication 2022 